Research Article

\title{
Remarks on Almost Cosymplectic 3-Manifolds with RICCI Operators
}

\author{
Quanxiang Pan (iD) ${ }^{1}$ and Yajie Wang ${ }^{2}$ \\ ${ }^{1}$ School of Science, Henan Institute of Technology, Henan 453003, China \\ ${ }^{2}$ School of Mathematics and Information Science, North China University of Water Resources and Electric Power, \\ Zhengzhou 450046, China \\ Correspondence should be addressed to Quanxiang Pan; panquanxiang@dlut.edu.cn
}

Received 28 December 2019; Accepted 15 February 2020; Published 16 March 2020

Academic Editor: Antonio Masiello

Copyright (c) 2020 Quanxiang Pan and Yajie Wang. This is an open access article distributed under the Creative Commons Attribution License, which permits unrestricted use, distribution, and reproduction in any medium, provided the original work is properly cited.

Let $M$ be an almost cosymplectic 3- $h$ - $a$-manifold. In this paper, we prove that the Ricci operator of $M$ is transversely Killing if and only if $M$ is locally isometric to a product space of an open interval and a surface of constant Gauss curvature, or a unimodular Lie group equipped with a left invariant almost cosymplectic structure. Some corollaries of this result and some examples illustrating main results are given.

\section{Introduction}

An almost cosymplectic manifold can be regarded as an odddimensional analogy of almost Kähler manifolds from topological points of view (see [1]) and was first introduced by Goldberg and Yano in [2]. In some recent literature, such kind of manifolds was also referred to as almost coKähler manifolds (see [3-9]); for some differences between these two types of manifolds, we refer the reader to a survey [1]. In this paper, we aim to investigate symmetry classification problems of almost cosymplectic manifolds and we adopt those notations and formulas introduced by Blair [10].

The curvature properties of cosymplectic manifolds were first studied by Blair who in [11] proved that a cosymplectic manifold of constant sectional curvature is locally flat. Olszak, in [12], extended this result and proved that an almost cosymplectic manifold of nonzero constant sectional curvature does not exist in dimensions $\geq 5$. Later, such a result was generalized to dimension three by Olszak in [13]. Perrone, in [14], extended Olszak's results and proved that a locally symmetric almost cosymplectic manifold of dimension three is cosymplectic and is locally isometric to a Riemannian product of a one-dimensional manifold and a Kähler surface of constant sectional curvature. Wang, in [5], studied locally symmetric almost cosymplectic manifolds and in particular, in [7], studied locally symmetric almost cosymplectic 5-manifolds. In recent years, many classification results on almost cosymplectic manifolds of dimension three emerged. For example, Cho, in [15], studied Reeb flow symmetry (that is, the Ricci tensor is invariant along the Reeb flow) on almost cosymplectic 3-manifolds. Moreover, semisymmetry, local $\phi$-symmetry, curvature, and ball homogeneities on almost cosymplectic 3-manifolds were considered in $[3,4,8,9]$, respectively. Some other symmetry properties in terms of the Ricci operators on such manifolds were also studied in $[6,16]$. For many symmetry properties on cosymplectic 3manifolds, we refer the reader to [17-19].

The notion of a Killing tensor field $T$ of type $(1,1)$ was introduced by Blair in [20], which is defined by

$$
\left(\nabla_{X} T\right) X=0,
$$

where $X$ and $\nabla$ denote an arbitrary vector field and the LeviCivita connection on a Riemannian manifold $(M, g)$, respectively. Relation (1) is much weaker than parallelism of Blair. In [20], Blair applied (1) for the structure tensor field to give a characterization for an almost contact metric manifold to be cosymplectic. In this paper, applying relation (1) for the Ricci operator, we obtain some local classification results of 
almost cosymplectic 3-manifolds and they are extensions of those in $[6,14]$.

\section{Almost Cosymplectic Manifolds}

By an almost contact metric manifold, we mean a Riemannian manifold $(M, g)$ of dimension $2 n+1$ on which there exists a quadruple $(\phi, \xi, \eta, g)$ satisfying

$$
\begin{aligned}
\phi^{2} & =-\mathrm{id}+\eta \otimes \xi, \\
\eta \circ \phi & =0, \\
\eta(\xi) & =1, \\
g(\phi X, \phi Y) & =g(X, Y)-\eta(X) \eta(Y),
\end{aligned}
$$

for any vector fields $X$ and $Y$, where $\phi$ is a $(1,1)$-type tensor field, $\xi$ is a vector field called the Reeb vector field, and $\eta$ is a global 1-form called the almost contact 1-form and $g$ is a Riemannian metric (see Blair [10]). By an almost cosymplectic manifold we mean an almost contact metric manifold on which there holds $\mathrm{d} \eta=0$ and $\mathrm{d} \Phi=0$, where $\Phi$ is the fundamental 2-form defined by $\Phi(X, Y)=g(X, \phi Y)$ (see $[10,12])$. We consider the product $M^{2 n+1} \times \mathbb{R}$ of an almost contact metric manifold $M^{2 n+1}$ and $\mathbb{R}$ and define on it an almost complex structure $J$ by

$$
J\left(X, f \frac{\mathrm{d}}{\mathrm{d} t}\right)=\left(\phi X-f \xi, \eta(X) \frac{\mathrm{d}}{\mathrm{d} t}\right),
$$

where $X$ denotes a vector field tangent to $M^{2 n+1}, t$ is the coordinate of $\mathbb{R}$, and $f$ is a $\mathscr{C}^{\infty}$-function on $M^{2 n+1} \times \mathbb{R}$. The almost contact metric manifold is said to be normal if $J$ is integrable, or equivalently,

$$
[\phi, \phi]=-2 \mathrm{~d} \eta \otimes \xi,
$$

where $[\phi, \phi]$ denotes the Nijenhuis tensor of $\phi$. A normal almost cosymplectic manifold is said to be a cosymplectic manifold (cf. [10]). An almost cosymplectic manifold is a cosymplectic manifold if and only if

$$
\nabla \phi=0 .
$$

Let $M^{2 n+1}$ be an almost cosymplectic manifold. We consider three tensor fields $l=R(\cdot, \xi) \xi, h=(1 / 2) \mathscr{L}_{\xi} \phi$, and $h^{\prime}=h \circ \phi$ on $M^{2 n+1}$, where $R$ is the Riemannian curvature tensor of $g$ and $\mathscr{L}$ is the Lie differentiation. From [10], we know that the three $(1,1)$-type tensor fields $l, h^{\prime}$, and $h$ are symmetric and satisfy

$$
\begin{aligned}
h \xi & =0, \\
l \xi & =0, \\
\operatorname{tr} h & =0, \\
\operatorname{tr}\left(h^{\prime}\right) & =0, \\
h \phi+\phi h & =0,
\end{aligned}
$$

and

$$
\nabla \xi=h^{\prime}
$$

\section{Main Results}

It is known that an almost cosymplectic manifold of dimension three is cosymplectic if and only if $h=0$ identically. Thus, we have to discuss our main theorems from two cases and first we consider the non-Kenmotsu case. Let $M^{3}$ be a three-dimensional almost cosymplectic manifold. Following Perrone [21], let $\mathcal{U}_{1}$ be the open subset of $M^{3}$ on which $h \neq 0$ and $\mathscr{U}_{2}$ the open subset defined by $\mathscr{U}_{2}=\left\{p \in M^{3}: h=0\right.$ in a neighborhood of $\left.p\right\}$. Therefore, $\mathcal{U}_{1} \cup \mathcal{U}_{2}$ is an open and dense subset of $M^{3}$. For any point $p \in \mathscr{U}_{1} \cup \mathscr{U}_{2}$, we find a local orthonormal basis $\left\{\xi, e_{1}, e_{2}=\phi e_{1}\right\}$ of three distinct unit eigenvector fields of $h$ in certain neighborhood of $p$. On $\mathcal{U}_{1}$, we assume that $h e_{1}=\lambda e_{1}$ and hence $h e_{2}=-\lambda e_{2}$, where $\lambda$ is assumed to be a positive function. Note that $\lambda$ is continuous on $M^{3}$ and smooth on $\mathscr{U}_{1} \cup \mathcal{U}_{2}$.

Lemma 1 (see [21]). On $\mathscr{U}_{1}$, we have

$$
\begin{aligned}
& \nabla_{\xi} e_{1}=a e_{2}, \\
& \nabla_{\xi} e_{2}=-a e_{1}, \\
& \nabla_{e_{1}} \xi=-\lambda e_{2}, \\
& \nabla_{e_{2}} \xi=-\lambda e_{1}, \\
& \nabla_{e_{1}} e_{1}=\frac{1}{2 \lambda}\left(e_{2}(\lambda)+\sigma\left(e_{1}\right)\right) e_{2}, \\
& \nabla_{e_{2}} e_{2}=\frac{1}{2 \lambda}\left(e_{1}(\lambda)+\sigma\left(e_{2}\right)\right) e_{1}, \\
& \nabla_{e_{2}} e_{1}=\lambda \xi-\frac{1}{2 \lambda}\left(e_{1}(\lambda)+\sigma\left(e_{2}\right)\right) e_{2}, \\
& \nabla_{e_{1}} e_{2}=\lambda \xi-\frac{1}{2 \lambda}\left(e_{2}(\lambda)+\sigma\left(e_{1}\right)\right) e_{1},
\end{aligned}
$$

where $f$ is a smooth function, $\sigma$ is the 1-form defined by $\sigma(\cdot)=S(\cdot, \xi)$, and $S$ is the Ricci tensor.

Applying Lemma 1, we obtain the Ricci operator $Q$ associated to the Ricci tensor given by (see [21])

$$
\begin{aligned}
Q= & \alpha \mathrm{id}+\beta \eta \otimes \xi+\phi \nabla_{\xi} h-\sigma\left(\phi^{2}\right) \otimes \xi+\sigma\left(e_{1}\right) \eta \otimes e_{1} \\
& +\sigma\left(e_{2}\right) \eta \otimes e_{2},
\end{aligned}
$$

where $\alpha=(1 / 2)\left(r+\operatorname{tr}\left(h^{2}\right)\right), \beta=-(1 / 2)\left(r+3 \operatorname{tr}\left(h^{2}\right)\right)$, and $r$ denotes the scalar curvature. Now, from (9), we write the Ricci operator as follows:

$$
\begin{aligned}
Q \xi & =-2 \lambda^{2} \xi+\sigma\left(e_{1}\right) e_{1}+\sigma\left(e_{2}\right) e_{2}, \\
Q e_{1} & =\sigma\left(e_{1}\right) \xi+\frac{1}{2}\left(r+2 \lambda^{2}-4 \lambda a\right) e_{1}+\xi(\lambda) e_{2}, \\
Q e_{2} & =\sigma\left(e_{2}\right) \xi+\xi(\lambda) e_{1}+\frac{1}{2}\left(r+2 \lambda^{2}+4 \lambda a\right) e_{2},
\end{aligned}
$$

with respect to the local orthonormal basis $\left\{\xi, e_{1}, e_{2}\right\}$. Moreover, according to Lemma 1 , by a simple calculation, we obtain 


$$
\nabla_{\xi} h=(1 / \lambda) \xi(\lambda) h-2 a h^{\prime} .
$$

In view of equation (11), Wang, in [6,9], considered a special almost coysmplectic 3-manifold determined by the condition $\nabla_{\xi} h=-2 a h^{\prime}$ with $a \in \mathbb{R}$. In this paper, we say that an almost cosymplectic 3-manifold satisfying the previous condition is an almost cosymplectic $3-h$ - $a$-manifold. In particular, when $a$ vanishes, an almost cosymplectic $3-h-a$ manifold reduces to an almost cosymplectic 3 - $h$-manifold (see [4]). Notice that on a cosymplectic manifold such a condition is meaningless because of $h=0$. There are many nontrivial examples of almost cosymplectic 3-manifolds satisfying such a condition even when $a$ is a smooth function (see $[4,6])$.

As seen in Section 1, the Ricci operator of an almost cosymplectic manifold is said to be transversely Killing if there holds

$$
\left(\nabla_{X} Q\right) X=0,
$$

for any vector field $X$ orthogonal to the Reeb vector field $\xi$. Obviously, (12) is much weaker than Ricci parallelism $(\nabla Q=0)$ and local symmetry $(\nabla R=0)$.

Now, we are ready to give our first main result.

Theorem 1. The Ricci operator of a noncosymplectic almost cosymplectic 3-h-a-manifold is transversely Killing if and only if the manifold is locally isometric to the group $E(2)$ of rigid motions of Euclidean 2-plane, the group $E(1,1)$ of rigid motions of Minkowski 2-plane, or the Heisenberg group $\mathrm{Nil}_{3}$ equipped with a left invariant almost cosymplectic structure.

Proof. On an almost cosymplectic 3- $h$-a-manifold, from the definition, we have $\nabla_{\xi} h=-2 a h^{\prime}$, and thus from (11), we may use $\xi(\lambda)=0$ in (10) to obtain

$$
\begin{gathered}
\mathrm{Q} \xi=-2 \lambda^{2} \xi+\sigma\left(e_{1}\right) e_{1}+\sigma\left(e_{2}\right) e_{2}, \\
Q e_{1}=\sigma\left(e_{1}\right) \xi+\frac{1}{2}\left(r+2 \lambda^{2}-4 \lambda a\right) e_{1}, \\
Q e_{2}=\sigma\left(e_{2}\right) \xi+\frac{1}{2}\left(r+2 \lambda^{2}+4 \lambda a\right) e_{2} .
\end{gathered}
$$

According to (13) and Lemma 1, by a standard calculation, we obtain

$$
\begin{aligned}
\left(\nabla_{e_{1}} Q\right) e_{1}= & \left(e_{1}\left(\sigma\left(e_{1}\right)\right)-\frac{1}{2 \lambda} \sigma\left(e_{2}\right)\left(e_{2}(\lambda)+\sigma\left(e_{1}\right)\right)\right) \xi \\
& +\frac{1}{2} e_{1}\left(r+2 \lambda^{2}-4 a \lambda\right) e_{1} \\
& -\left(\lambda \sigma\left(e_{1}\right)+2 a\left(e_{2}(\lambda)+\sigma\left(e_{1}\right)\right)\right) e_{2}, \\
\left(\nabla_{e_{2}} Q\right) e_{2}= & \left(e_{2}\left(\sigma\left(e_{2}\right)\right)-\frac{1}{2 \lambda} \sigma\left(e_{1}\right)\left(e_{1}(\lambda)+\sigma\left(e_{2}\right)\right)\right) \xi \\
& -\left(\lambda \sigma\left(e_{2}\right)-2 a\left(e_{1}(\lambda)+\sigma\left(e_{2}\right)\right)\right) e_{1} \\
& +\frac{1}{2} e_{2}\left(r+2 \lambda^{2}+4 a \lambda\right) e_{2} .
\end{aligned}
$$

Because the Ricci operator is transversely Killing, from (12), we have $\left(\nabla_{e_{1}} Q\right) e_{1}=0$ and $\left(\nabla_{e_{2}} Q\right) e_{2}=0$, which are compared with (14) and (15), respectively, implying

$$
\begin{aligned}
e_{1}\left(\sigma\left(e_{1}\right)\right)-\frac{1}{2 \lambda} \sigma\left(e_{2}\right)\left(e_{2}(\lambda)+\sigma\left(e_{1}\right)\right) & =0, \\
e_{1}\left(r+2 \lambda^{2}-4 a \lambda\right) & =0, \\
\lambda \sigma\left(e_{1}\right)+2 a\left(e_{2}(\lambda)+\sigma\left(e_{1}\right)\right) & =0,
\end{aligned}
$$

and

$$
\begin{aligned}
e_{2}\left(\sigma\left(e_{2}\right)\right)-\frac{1}{2 \lambda} \sigma\left(e_{1}\right)\left(e_{1}(\lambda)+\sigma\left(e_{2}\right)\right) & =0, \\
\lambda \sigma\left(e_{2}\right)-2 a\left(e_{1}(\lambda)+\sigma\left(e_{2}\right)\right) & =0, \\
e_{2}\left(r+2 \lambda^{2}+4 a \lambda\right) & =0,
\end{aligned}
$$

respectively. On the contrary, applying Lemma 1, we also have

$$
\begin{aligned}
{\left[\xi, e_{1}\right] } & =(a+\lambda) e_{2}, \\
{\left[e_{2}, \xi\right] } & =(a-\lambda) e_{1}, \\
{\left[e_{1}, e_{2}\right] } & =\frac{1}{2 \lambda}\left(e_{1}(\lambda)+\sigma\left(e_{2}\right)\right) e_{2}-\frac{1}{2 \lambda}\left(e_{2}(\lambda)+\sigma\left(e_{1}\right)\right) e_{1} .
\end{aligned}
$$

Putting (18) into the well-known Jacobi identity, we obtain

$$
\begin{aligned}
& e_{1}(\lambda-a)+\xi\left(\frac{e_{2}(\lambda)+\sigma\left(e_{1}\right)}{2 \lambda}\right)+\frac{a-\lambda}{2 \lambda}\left(e_{1}(\lambda)+\sigma\left(e_{2}\right)\right)=0, \\
& e_{2}(\lambda+a)+\xi\left(\frac{e_{1}(\lambda)+\sigma\left(e_{2}\right)}{2 \lambda}\right)-\frac{a+\lambda}{2 \lambda}\left(e_{2}(\lambda)+\sigma\left(e_{1}\right)\right)=0 .
\end{aligned}
$$

Recall that, in this situation, $a \in \mathbb{R}$ and $\xi(\lambda)=0$ and the abovementioned relations reduce to

$$
\begin{aligned}
& e_{1}(\lambda)+\frac{1}{2 \lambda} \xi\left(e_{2}(\lambda)+\sigma\left(e_{1}\right)\right)+\frac{a-\lambda}{2 \lambda}\left(e_{1}(\lambda)+\sigma\left(e_{2}\right)\right)=0, \\
& e_{2}(\lambda)+\frac{1}{2 \lambda} \xi\left(e_{1}(\lambda)+\sigma\left(e_{2}\right)\right)-\frac{a+\lambda}{2 \lambda}\left(e_{2}(\lambda)+\sigma\left(e_{1}\right)\right)=0 .
\end{aligned}
$$

Taking into account the last term of (16) (or the second term of (17)), we have to divide our discussions into the following two cases.

Case 1. $a=0$. Because $\lambda$ is assumed to be a positive function, using $a=0$ in the last term of (16) and the second term of (17), we obtain

$$
\sigma\left(e_{1}\right)=\sigma\left(e_{2}\right)=0 .
$$

As $\xi(\lambda)=0$ and $a=0$, from the first two terms of (18), we obtain $\xi\left(e_{1}(\lambda)\right)=\lambda e_{2}(\lambda)$ and $\xi\left(e_{2}(\lambda)\right)=\lambda e_{1}(\lambda)$. Putting 
these two equations into (20), with the aid of (21) and $a=0$, we obtain

$$
e_{1}(\lambda)=e_{2}(\lambda)=0
$$

where we have used that $\lambda$ is a positive function. In view of $\xi(\lambda)=0$, together with the abovementioned relations, we see that $\lambda$ is a positive constant. Now (18) reduces to

$$
\begin{aligned}
{\left[\xi, e_{1}\right] } & =\lambda e_{2}, \\
{\left[e_{2}, \xi\right] } & =-\lambda e_{1}, \\
{\left[e_{1}, e_{2}\right] } & =0 .
\end{aligned}
$$

According to Milnor [22] and the abovementioned relations, it is easily seen that the manifold is locally isometric to the group $E(1,1)$ of rigid motions of the Minkowski 2space equipped with a left invariant almost cosymplectic structure.

Case 2. $a \neq 0$. In this case, replacing $X$ by $e_{1}+e_{2}$ in (12), with the aid of $\left(\nabla_{e_{1}} Q\right) e_{1}=0$ and $\left(\nabla_{e_{1}} Q\right) e_{1}=0$, we obtain

$$
\left(\nabla_{e_{1}} Q\right) e_{2}+\left(\nabla_{e_{2}} Q\right) e_{1}=0
$$

On the contrary, with the aid of $\xi(\lambda)=0$, applying (13) and Lemma 1, we obtain

$$
\begin{aligned}
& \left(\nabla_{e_{1}} Q\right) e_{2}=\left(e_{1}\left(\sigma\left(e_{2}\right)\right)+\frac{1}{2} \lambda\left(r+4 a \lambda+6 \lambda^{2}\right)+\frac{1}{2 \lambda} \sigma\left(e_{1}\right)\left(e_{2}(\lambda)+\sigma\left(e_{1}\right)\right)\right) \xi-\left(\lambda \sigma\left(e_{1}\right)+2 a\left(e_{2}(\lambda)+\sigma\left(e_{1}\right)\right)\right) e_{1} \\
& -\left(2 \lambda \sigma\left(e_{2}\right)-\frac{1}{2} e_{1}\left(r+2 \lambda^{2}+4 a \lambda\right)\right) e_{2} \\
& -\left(\lambda \sigma\left(e_{2}\right)-2 a\left(e_{1}(\lambda)+\sigma\left(e_{2}\right)\right)\right) e_{2} \\
& e_{1}\left(\sigma\left(e_{2}\right)\right)+e_{2}\left(\sigma\left(e_{1}\right)\right)+\lambda\left(r+6 \lambda^{2}\right)+\frac{1}{2 \lambda} \sigma\left(e_{2}\right)\left(e_{1}(\lambda)+\sigma\left(e_{2}\right)\right) \\
& +\frac{1}{2 \lambda} \sigma\left(e_{1}\right)\left(e_{2}(\lambda)+\sigma\left(e_{1}\right)\right)=0 \\
& 3 \lambda \sigma\left(e_{1}\right)+2 a\left(e_{2}(\lambda)+\sigma\left(e_{1}\right)\right)-\frac{1}{2} e_{2}\left(r+2 \lambda^{2}-4 a \lambda\right)=0, \\
& 3 \lambda \sigma\left(e_{2}\right)-2 a\left(e_{1}(\lambda)+\sigma\left(e_{2}\right)\right)-\frac{1}{2} e_{1}\left(r+2 \lambda^{2}+4 a \lambda\right)=0 .
\end{aligned}
$$$$
\left(\nabla_{e_{2}} Q\right) e_{1}=\left(e_{2}\left(\sigma\left(e_{1}\right)\right)+\frac{1}{2} \lambda\left(r-4 a \lambda+6 \lambda^{2}\right)+\frac{1}{2 \lambda} \sigma\left(e_{2}\right)\left(e_{1}(\lambda)+\sigma\left(e_{2}\right)\right)\right) \xi-\left(2 \lambda \sigma\left(e_{1}\right)-\frac{1}{2} e_{2}\left(r+2 \lambda^{2}-4 a \lambda\right)\right) e_{1}
$$

Putting the last term of (17) and the second term of (16) into (27) and (28), respectively, we obtain

$$
(3 \lambda+2 a) \sigma\left(e_{1}\right)+6 a e_{2}(\lambda)=0,
$$

and

$$
(3 \lambda-2 a) \sigma\left(e_{2}\right)-6 a e_{1}(\lambda)=0,
$$

respectively. The subtraction of (29) from the third term of (16) multiplied by 3 implies $\sigma\left(e_{1}\right)=0$, where we have used the assumption $a \neq 0$. Using this relation back in (29), we obtain

$$
e_{2}(\lambda)=0
$$

Similarly, in view of $a \neq 0$, the subtraction of (30) from the second term of (17) multiplied by 3 gives $\sigma\left(e_{2}\right)=0$, which is applied back in (30), implying

$$
e_{1}(\lambda)=0
$$

In view of $\xi(\lambda)=0$, we see that $\lambda$ is a positive constant. Moreover, from (26), we see that the scalar curvature of the manifold is a negative constant. In this case, from (18), we also have

$$
\begin{aligned}
{\left[\xi, e_{1}\right] } & =(a+\lambda) e_{2}, \\
{\left[e_{2}, \xi\right] } & =(a-\lambda) e_{1}, \\
{\left[e_{1}, e_{2}\right] } & =0 .
\end{aligned}
$$

Finally, according to Milnor [22] and (33), we observe that the manifold is locally isometric to a unimodular Lie group $G$ endowed with a left invariant noncosymplectic almost cosymplectic structure because

$$
\begin{array}{r}
\operatorname{trace}\left\{e_{i} \longrightarrow\left[e_{i}, e_{j}\right]\right\}=0, \\
\text { for any }\left\{e_{1}, e_{2}, e_{3}=\xi\right\} .
\end{array}
$$

More specifically, $G$ is the group $E(2)$ of rigid motions of Euclidean 2-plane if $a>\lambda$ or $a<-\lambda$; the group $E(1,1)$ of rigid motions of Minkowski 2-plane if $-\lambda<a<\lambda$; or the Heisenberg group $\mathrm{Nil}_{3}$ if $a=\lambda$ or $a=-\lambda$. We refer the reader to [14] for the constructions of almost cosymplectic structures on the abovementioned unimodular Lie groups. The converse is easy to check. This completes the proof.

From proof of Theorem 1, we have

Corollary 1. The Ricci operator of a noncosymplectic almost cosymplectic 3-h-manifold is transversely Killing if and only if the manifold is locally isometric to the group $E(1,1)$ of rigid motions of Minkowski 2-plane equipped with a left invariant almost cosymplectic structure. 
In Theorem 1, the condition "the Ricci operator is transversely Killing" is essential because we have

Example 1. Let $G$ be a three-dimensional nonunimodular Lie group equipped with a left invariant metric $g$ whose Lie algebra is given by

$$
\begin{aligned}
& {\left[e_{1}, e_{2}\right]=\alpha e_{2},} \\
& {\left[e_{2}, e_{3}\right]=0,} \\
& {\left[e_{1}, e_{3}\right]=\beta e_{2},}
\end{aligned}
$$

where $\left\{e_{1}, e_{2}, e_{3}\right\}$ is an orthonomal basis with respect to $g$ and $\alpha, \beta \in \mathbb{R}^{*}$. On $G$, there exists an almost cosymplectic structure (see [14]). According to [4], we have

$$
\begin{aligned}
& \left(\nabla_{e_{1}} Q\right) e_{1}=0 \\
& \left(\nabla_{e_{2}} Q\right) e_{2}=\frac{1}{2} \alpha \beta^{2} e_{1} .
\end{aligned}
$$

It is easily seen that the left-invariant Ricci operator of $G$ is not transversely Killing even if $\nabla_{\xi} h=\beta h$. follows.

Next, we give the cosymplectic version of Theorem 1 as

Theorem 2. The Ricci operator of a cosymplectic 3-manifold is transversely Killing if and only if the manifold is locally isometric to a Riemannian product of an open interval and a surface of constant Gauss curvature.

Proof. On a cosymplectic 3-manifold, applying $h=0$ on (7), we see that $\xi$ is parallel, and hence we get $R(X, Y) \xi=0$ and

$$
Q \xi=0 .
$$

On a Riemannian 3-manifold, the curvature tensor $R$ is given by

$$
\begin{aligned}
R(X, Y) Z= & g(Y, Z) Q X-g(X, Z) Q Y+g(Q Y, Z) X \\
& -g(Q X, Z) Y-\frac{r}{2}(g(Y, Z) X-g(X, Z) Y),
\end{aligned}
$$

for any vector fields $X, Y$, and $Z$, where $r$ is the scalar curvature. Replacing $Z$ by $\xi$ in (38), with the aid of (37) and $R(X, Y) \xi=0$, we obtain

$$
Q X=\frac{r}{2} X-\frac{r}{2} \eta(X) \xi,
$$

for any vector field $X$. According to (7) and (37), a simple calculation shows $\left(\nabla_{\xi} Q\right) \xi=0$. If the Ricci operator is transversely Killing, from (12), we obtain $\left(\nabla_{e} Q\right) e=0$ and $\left(\nabla_{\phi e} Q\right) \phi e=0$, where $\{e, \phi e\}$ is an arbitrary local basis of the contact distribution $\{\xi\}^{\perp}$. Thus, applying the previous three relations in the well-known formula $\operatorname{div} Q=1 / 2 \operatorname{grad} r$, we obtain that the scalar curvature $r$ is a constant. Therefore, taking the derivative of (39), we obtain

$$
\nabla Q=0 .
$$

That is, the manifold is locally symmetric, and hence following [14], we see that the manifold is locally isometric to a product space of an open interval and a surface of constant Gauss curvature. The converse is easy to check.

An almost cosymplectic 3-manifold is said to be an almost cosymplectic 3 - $h$-manifold if the Reeb vector field $\xi$ is principal direction of the Ricci operator, or equivalently, $\xi$ is harmonic (see [4]).

Lemma 2. If on an almost cosymplectic 3-h-manifold $M$, the Ricci operator is transversely Killing, then we have $\xi(\lambda)=0$ and $d a \wedge \eta=0$.

Proof. If $\xi$ is harmonic, we have $Q \xi=S(\xi, \xi) \xi$, which is applied in Lemma 1 implying $\sigma\left(e_{1}\right)=0$ and $\sigma\left(e_{2}\right)=0$. Using this, now (10) becomes

$$
\begin{aligned}
Q \xi & =-2 \lambda^{2} \xi, \\
Q e_{1} & =\frac{1}{2}\left(r+2 \lambda^{2}-4 \lambda a\right) e_{1}+\xi(\lambda) e_{2}, \\
Q e_{2} & =\xi(\lambda) e_{1}+\frac{1}{2}\left(r+2 \lambda^{2}+4 \lambda a\right) e_{2} .
\end{aligned}
$$

Taking into account Lemma 1 and (41), we obtain

$$
\begin{aligned}
\left(\nabla_{e_{1}} Q\right) e_{1}= & \lambda \xi(\lambda) \xi+\left(e_{1}(\xi(\lambda))-2 a e_{2}(\lambda)\right) e_{2} \\
& +\left(\frac{1}{2} e_{1}\left(r+2 \lambda^{2}-4 \lambda a\right)-\frac{1}{\lambda} \xi(\lambda) e_{2}(\lambda)\right) e_{1},
\end{aligned}
$$

and

$$
\begin{aligned}
\left(\nabla_{e_{2}} Q\right) e_{2}= & \lambda \xi(\lambda) \xi+\left(e_{2}(\xi(\lambda))+2 a e_{1}(\lambda)\right) e_{1} \\
& +\left(\frac{1}{2} e_{2}\left(r+2 \lambda^{2}+4 \lambda a\right)-\frac{1}{\lambda} \xi(\lambda) e_{1}(\lambda)\right) e_{2} .
\end{aligned}
$$

If the Ricci operator is transversely Killing, from (12), we have $\left(\nabla_{e_{1}} Q\right) e_{1}=0$ and $\left(\nabla_{e_{2}} Q\right) e_{2}=0$, which are compared with (42) and (43), implying

$$
\begin{aligned}
\xi(\lambda) & =0, \\
a e_{2}(\lambda) & =0, \\
e_{1}\left(r+2 \lambda^{2}-4 a \lambda\right) & =0,
\end{aligned}
$$

and

$$
\begin{aligned}
\xi(\lambda) & =0, \\
a e_{1}(\lambda) & =0, \\
e_{2}\left(r+2 \lambda^{2}+4 a \lambda\right) & =0,
\end{aligned}
$$

respectively, where we have applied that $\lambda$ is a positive function. If $a$ vanishes, the proof is completed already. Next we consider the case that $a \neq 0$ on some open subset. In this case, from (44) and (45), we know that $\lambda$ is a positive constant. Moreover, from Lemma 1 and (41), we obtain 


$$
\left(\nabla_{\xi} Q\right) \xi=0
$$

Applying (46), $\left(\nabla_{e_{1}} Q\right) e_{1}=0$, and $\left(\nabla_{e_{2}} Q\right) e_{2}=0$ in the formula $\operatorname{div} Q=1 / 2 \operatorname{grad} r$, we see that $r$ is also a constant. Thus, according to (44) and (45), we obtain

$$
e_{1}(a)=e_{2}(a)=0 \text {. }
$$

It follows directly that $\mathrm{d} a=\xi(a) \eta$ and hence by the definition of almost cosymplectic structure we get $\mathrm{d} a \wedge \eta=0$. This completes the proof.

Theorem 3. Let $M$ be a noncosymplectic almost cosymplectic 3-h-manifold such that $a$ is an invariant along Reeb flow. Then, the Ricci operator is transversely Killing if and only if the manifold is locally isometric to the group E(2) of rigid motions of Euclidean 2-plane, the group $E(1,1)$ of rigid motions of Minkowski 2-plane, or the Heisenberg group $\mathrm{Nil}_{3}$ equipped with a left invariant almost cosymplectic structure.

Proof. On a noncosymplectic almost cosymplectic 3- $h$ manifold $M$, if $a$ is an invariant along the Reeb flow, from Lemma 2, we see that $a$ is a constant. Thus, together with $\xi(\lambda)=0$, we see that $M$ is in fact an almost cosymplectic 3- $h$ $a$-manifold. Finally, the proof follows immediately from Theorem 1.

Note that the condition " $a$ is invariant along the Reeb flow and (12) is true" is essential. We show an almost cosymplectic 3 - $h$-manifold on which previous condition does not hold (see $[4,23]$ ).

Example 2. Let $M^{3}$ be an open subset of $\mathbb{R}^{3}$ defined by $M^{3}:=\left\{(x, y, z) \in \mathbb{R}^{3}: z>0\right\}$. On $M^{3}$ there is an almost cosymplectic structure defined by

$$
\begin{aligned}
\xi & =\frac{\partial}{\partial z}, \\
\eta & =\mathrm{d} z, \\
\phi \frac{\partial}{\partial x} & =z^{2} \frac{\partial}{\partial y} \\
\phi \frac{\partial}{\partial y} & =-\frac{1}{z^{2}} \frac{\partial}{\partial x}, \\
\phi \frac{\partial}{\partial z} & =0, \\
g & =z^{2} \mathrm{~d} x \otimes \mathrm{d} x+\frac{1}{z^{2}} \mathrm{~d} y \otimes \mathrm{d} y+\mathrm{d} z \otimes \mathrm{d} z .
\end{aligned}
$$

An orthonormal basis for the tangent space at each point of $M$ is given by $\left\{\xi, e_{1}=(1 / z)(\partial / \partial x), e_{2}=z(\partial / \partial y)\right\}$. According to [4], we have

$$
\begin{aligned}
Q \xi & =-\frac{2}{z^{2}} \xi \\
\left(\nabla_{e_{1}} Q\right) e_{1} & =-\frac{3}{z^{2}} \xi \\
\left(\nabla_{e_{1}} Q\right) e_{2}= & \left(\nabla_{e_{2}} Q\right) e_{1}=0 \\
\left(\nabla_{e_{2}} Q\right) e_{2} & =\frac{1}{z^{2}} \xi
\end{aligned}
$$

From this, we see that the Reeb vector field is harmonic but the Ricci operator is not transversely Killing.

\section{Data Availability}

The data used to support the findings of this study are available from the corresponding author upon request.

\section{Conflicts of Interest}

The authors declare that they have no conflicts of interest.

\section{Acknowledgments}

This paper was supported by the Doctoral Foundation of Henan Institute of Technology (grant no. KQ1828).

\section{References}

[1] B. Cappelletti-Montano, A. De Nicola, and I. Yudin, "A survey on cosymplectic geometry," Reviews in Mathematical Physics, vol. 25, no. 10, Article ID 1343002, 55 pages, 2013.

[2] S. Goldberg and K. Yano, "Integrability of almost cosymplectic structures," Pacific Journal of Mathematics, vol. 31, no. 2, pp. 373-382, 1969.

[3] U. C. De, P. Majhi, and Y. J. Suh, "Semi-symmetric properties of almost coKähler 3-manifolds," Bulletin of the Korean Mathematical Society, vol. 56, pp. 219-228, 2019.

[4] X. Liu and W. Wang, "Locally $\phi$-symmetric almost coKähler 3-manifolds," Bulletin Mathematique de la Societe des Sciences Mathematiques de Roumanie, vol. 62, pp. 427-438, 2019.

[5] Y. Wang, "Almost co-Kähler manifolds satisfying some symmetry conditions," Turkish Journal of Mathematics, vol. 40, pp. 740-752, 2016.

[6] Y. Wang, "Ricci tensors on three-dimensional almost coKähler manifolds," Kodai Mathematical Journal, vol. 39, no. 3, pp. 469-483, 2016.

[7] Y. Wang, "Locally symmetric almost coKähler 5-manifolds with Kählerian leaves," Bulletin of the Korean Mathematical Society, vol. 55, pp. 789-798, 2018.

[8] Y. Wang, "Semi-symmetric almost coKähker 3-manifolds," International Journal of Geometric Methods in Modern Physics, vol. 15, Article ID 1850031, 12 pages, 2018.

[9] Y. Wang, "Curvature homogeneity and ball-homogeneity on almost coKähler 3-manifolds," Bulletin of the Korean Mathematical Society, vol. 56, pp. 253-263, 2019.

[10] D. E. Blair, "Riemannian geometry of contact and symplectic manifolds," Progress in Mathematics, vol. 203, 2010. 
[11] D. E. Blair, "The theory of quasi-Sasakian structures," Journal of Differential Geometry, vol. 1, no. 3-4, pp. 331-345, 1967.

[12] Z. Olszak, "On almost cosymplectic manifolds," Kodai Mathematical Journal, vol. 4, no. 2, pp. 239-250, 1981.

[13] Z. Olszak, "Almost cosymplectic manifolds with Kählerian leaves,” Tensor (N. S.), vol. 46, pp. 117-124, 1987.

[14] D. Perrone, "Classification of homogeneous almost cosymplectic three-manifolds," Differential Geometry and Its Applications, vol. 30, no. 1, pp. 49-58, 2012.

[15] J. T. Cho, "Reeb flow symmetry on almost cosymplectic threemanifolds," Bulletin of the Korean Mathematical Society, vol. 53, no. 4, pp. 1249-1255, 2016.

[16] W. Wang and X. Liu, "Three-dimensional almost coKähler manifolds with harmonic Reeb vector fields," Revista de la Union Matematica Argentina, vol. 58, pp. 307-317, 2017.

[17] U. C. De and A. K. Sengupta, "Notes on three-dimensional quasi-Sasakian manifolds," Demonstratio Mathematica, vol. 37, no. 3, pp. 655-660, 2004.

[18] U. C. De, A. Yildiz, and A. Çetinkaya, "Certain results on a type of contact metric manifold," Afrika Matematika, vol. 26, no. 2-15, pp. 1229-1236, 2015.

[19] U. C. De, A. Yildiz, and A. Yalınız, "Locally $\phi$-symmetric normal almost contact metric manifolds of dimension 3," Applied Mathematics Letters, vol. 22, no. 5, pp. 723-727, 2009.

[20] D. Blair, "Almost contact manifolds with Killing structure tensors," Pacific Journal of Mathematics, vol. 39, no. 2, pp. 285-292, 1971.

[21] D. Perrone, "Minimal Reeb vector fields on almost cosymplectic manifolds," Kodai Mathematical Journal, vol. 36, no. 2, pp. 258-274, 2013.

[22] J. Milnor, "Curvatures of left invariant metrics on lie groups," Advances in Mathematics, vol. 21, no. 3, pp. 293-329, 1976.

[23] P. Dacko and Z. Olszak, "On conformally almost cosymplectic manifolds with Kählerian leaves," Rendiconti del Seminario Matematico. Università e Politecnico Torino, vol. 56, pp. 89103, 1998. 